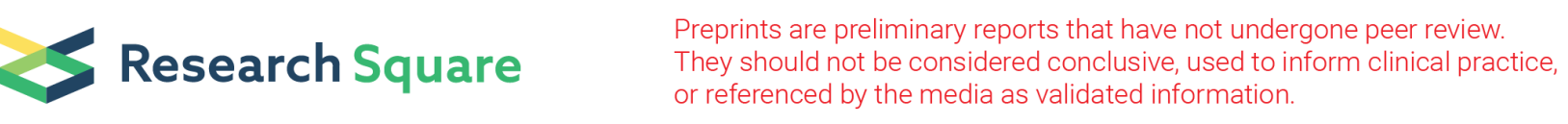

\title{
A novo missense variant of the PHEX gene in patients with X-linked dominant hypophosphatemia
}

peixuan cao ( $\square$ caopeixuan1993@126.com )

Nanjing University Medical School Affiliated Nanjing Drum Tower Hospital https://orcid.org/00000002-4779-5512

\section{Xiangyu Zhu}

Nanjing University Medical School Affiliated Nanjing Drum Tower Hospital

\section{Leilei Gu}

Nanjing University Medical School Affiliated Nanjing Drum Tower Hospital

Wei Liu

Nanjing University Medical School Affiliated Nanjing Drum Tower Hospital Jie Li

Nanjing University Medical School Affiliated Nanjing Drum Tower Hospital

\section{Research}

Keywords: Genetic diagnosis, Missense variant, Phosphate-regulating gene, X-linked hypophosphatemia

Posted Date: April 1st, 2020

DOI: https://doi.org/10.21203/rs.3.rs-19833/v1

License: (c) (i) This work is licensed under a Creative Commons Attribution 4.0 International License. Read Full License 


\section{Abstract}

X-linked hypophosphatemia (XLH) is characterized by low serum phosphate concentration. Both males and females could be affected, and males tend to perform more severely. Identification of a pathological variation in the phosphate-regulating gene with homologies to endopeptidase on the $\mathrm{X}$ chromosome ( PHEX ) gene regulating phosphate located in the $X$ chromosome by molecular genetic detection confirms the diagnosis. The current pharmacologic treatments mainly focus on relieving symptoms instead of preventing it occur. A maternally inherited novo missense heterozygous variant c.1256G>A in exon 11 of the PHEX gene was found in the proband and her mother. SIFT, Polyphen2 and PROVEAN predicted it as a deleterious mutation. This mutation was also detected in an affected maternal grandmother and an affected maternal uncle, a healthy maternal uncle and three healthy maternal aunts and their offspring did not carry this mutation. We identified a novo PHEX variant c.1256G>A, p.(Gly419Glu), the heterozygous mutation may be the cause of the deformity in this family.

\section{Introduction}

X-linked hypophosphatemia (XLH, OMIM 307800) is the most common hereditary metabolic rickets with a prevalence of 1:20,000 [1]. A spectrum of phenotypes ranging from isolated hypophosphatemia to severe bone deformities is observed in patients of XLH. Comparing to upper extremities, lower extremities are always more extensively involved and severe in XLH patients, especially lower-extremity bowing. In adults with XLH, short stature and impaired mobility may be the only reason seeking for help. Besides, researchers also found individuals with XLH are prone to spontaneous dental abscesses, sensorineural hearing loss could occur in XLH patients [2]. XLH is transmitted in X-linked dominant fashion. Though there is no known difference in penetrance between males and females, males usually present more severe symptoms. Children are usually treated in pharmacology, however, in adults, the role of medicine is less clear, surgery could be required if patients with severe symptoms.

The mutation in the phosphate-regulating gene with homologies to endopeptidase on the $\mathrm{X}$ chromosome (PHEX) was firstly described in 1995 as the causative gene for XLH [3]. Based on the Human Gene Mutation database (HGMD professional 2018.4release), a total of 457 different PHEX mutations have been identified until now [4], including missense variants, nonsense variants, splicing variants, and insertion variants. However, no hot spot mutation in PHEX gene has been reported. As no efficient treatment exits and its highly disabling, molecular genetic testing is vital to confirm the diagnosis and prevent the occurrence of XLH.

In this study, we reported a Chinese family with $4 \mathrm{XLH}$ patients, and found a novo missense mutation (c.1256G > A) on one allele of the PHEX gene, this mutation may be responsible for the XLH family.

\section{Methods}

\section{Ethics approval}


This study was approved by the Ethics Committee of the Drum Tower Hospital, Nanjing University (Jiangsu, China). Informed consent was given by the family (Fig. 1), who agreed to joining this study and the use of the clinical data for scientific research and publication. The study was performed in the Prenatal Diagnosis Center of Nanjing Drum Tower Hospital (Jiangsu, China). The methods used in this study were performed in accordance with the approved guidelines.

\section{Clinical Features}

The proband was a 20-year-old Chinese woman (囚-5), she was short in stature (150 cm), physical examination showed no obvious symptoms except lower-extremity bowing (valgus deformities). She did not have musculoskeletal complaints, such as stress fractures, joint pain or osteomalacia, she could walk, run and jump without impaired mobility. Besides, her teeth and hearing were both normal, no dental abscesses or sensorineural hearing loss was found. Her intelligence and development were no different from healthy females. No medical treatment had been offered before. The X-ray of her lower extremities indicated knock knees, and a bilateral bowed femur, tibia and fibula (Fig. 2). The laboratory tests showed a low serum phosphate level $(0.73 \mathrm{mmol} / \mathrm{I})$, normal serum calcium level $(2.3 \mathrm{mmol} / \mathrm{I})$ and normal serum parathyroid hormone $(22.33 \mathrm{pg} / \mathrm{ml})$. Her family history was remarkable. The proband's father ( $\mathbb{1}-10)$ was healthy, but her mother ( $₫-9)$ had similar symptoms, such as short stature $(145 \mathrm{~cm})$ and lower-extremity bowing (valgus deformities). The proband had three maternal aunts and two maternal uncles, except a maternal uncle ( $(-11)$ with similar and more severe symptoms, the other maternal uncle, aunts and their

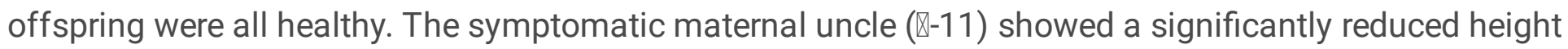
$(135 \mathrm{~cm})$ comparing to reference standards and more serious lower-extremity bowing (valgus deformities). In addition, joint pain and impair mobility had been occurred. The proband's maternal

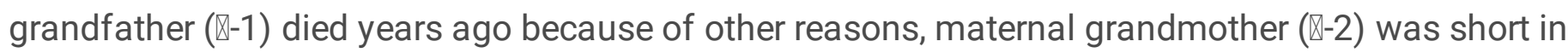
stature with lower-extremity bowing (valgus deformities). The laboratory tests and X-ray images were all lacked in the affected family members except proband.

\section{DNA Extraction}

Genomic DNA was extracted from peripheral whole blood samples, using the QIAamp DNA Blood kit (Qiagen, Hilden, Germany) according to the manufacturer's protocol. DNA was stored at $-20^{\circ} \mathrm{C}$ prior to use.

\section{Trio-based Whole-exome Sequencing}

Collected the peripheral whole blood samples from proband and her parents, sent blood samples to Berry Genomics Corporation (Beijing, China) to perform the trio-based whole-exome sequencing (WES), and the BWA (Burrowa-Wheeler, v.0.5.9-r16) alignment algorithm was used to compare with the UCSC(http://genome.ucsc.edu/) hg19 human reference genome sequence, and the GATK (Genomic Analysis Tool Kit) was used to identify the mutation. To predict the outcomes of variants, the Human 
Gene Variant database (http://www.hgmd.cf.ac.uk), ExAC database, 1000 Genome database were used to annotate the detected mutation sites, and SIFT (https://sift.bii.a-star.edu.sg/), PolyPhen2 (http://genetics.bwh.harvard.edu/pph2/) and other software were used to predict the effect of the nucleotide changes on protein function.

\section{Sanger Sequencing Validation}

A variant of the PHEX gene was identified in proband and her mother, the detected variants were confirmed using PCR. Information of the PHEX gene was obtained from the online NCBI database (https://www.ncbi.nlm.nih.gov/pubmed; RefSeq NM_000444.5). 1 pair of primer was designed for PCR amplification of the exon region that contains mutation and intron/exon boundaries of the PHEX gene using an online tool (http://www.primer3.com/). The following primer was used for a 582-base pair (bp) target sequence in exon 11: F:5'-CACTTTGACCGGCCTAAAAC-3', R:5'-CCCCTGGAAAACTACACACC-3'. The PCR conditions were as follows: $94^{\circ} \mathrm{C}$ for $3 \mathrm{~min}, 94^{\circ} \mathrm{C}$ for $30 \mathrm{sec}, 57^{\circ} \mathrm{C}$ for $30 \mathrm{sec}, 68^{\circ} \mathrm{C}$ for $15 \mathrm{sec}$, (39 cycles) and $68^{\circ} \mathrm{C}$ for $6 \mathrm{~min}, 4^{\circ} \mathrm{C}$ forever. The PCR-amplified DNA products were ectrophoresed on a $2 \%$ agarose gel, then were submitted to sanger sequencing in Sangon Biotech Co., Ltd. (Shanghai, China). Sequencing results were analyzed using Seqman software in the DNA star software package and aligned with the UCSC hg19 human standard sequence.

\section{Results}

\section{Identification of mutations in PHEX in the trio}

By trio-based whole-exome sequencing of the trio, we identified a novo variant in PHEX gene (NM_000444.5: c.1256G > A) in proband and her mother.

\section{Pathogenicity Analysis Of Variants}

In the trio, the variant in PHEX gene resulted in the Glycine changing to Glutamic acid (p. Gly419Glu). The variant site in 7 species is highly conserved (Fig. 3), showing its important role in the function of the protein. This variant was absent in public databases including the gnomAD, HGVD, GenoAD and 1000G. This substitution was predicted to be deleterious by multiple in silico pathogenicity prediction tools: CADD PHRED score was 27.9 (http://cadd.gs.washington.edu/snv), PP2_HVAR score was 0.996 (http://genetics.bwh.harvard.edu/pph2/), PROVEAN score was - 7.67(http://provean.jcvi.org/) and SIFT score was 0 (http://sift.jcvi.org/). Therefore, the p.Gly419Glu variant is very likely to affect function of PHEX.

\section{Variants Verification Of Pedigree Members}


The PHEX gene c.1256G > A variant was found in a symptomatic maternal uncle and maternal grandmother, not found in healthy family members. The sequencing results of the family members showing the variant: In the male patient (II11), there is a only peak (A). However, in female patients (I2, II9, $\varangle 5$ ), there are heterozygous peaks (A/G) (Fig. 4).

\section{Discussion}

This study reported a family with XLH, which is a metabolic disease through the whole life. The most obvious feature of XLH is short stature and deformities of both lower limbs. The height of the patients is shorter than the reference standards [5]. With the accumulation of weight bearing, lower-extremity bowing becomes evident, even joint pain and impaired mobility could occur at any age. Dental abnormalities such as spontaneous dental abscesses have been reported, which resulting the teeth dentin component changing. Hearing loss could also present in XLH patients. The gold standard is identifying the pathogenic variant in the proband by molecular genetic testing.

PHEX gene, which is the only causative gene for XLH. Pathogenic variants in PHEX lead to increased serum levels of fibroblast growth factor 23 (FGF23) [6]. As there is no direct link between PHEX and FGF23, the molecular mechanism is not clear. FGF23 controls phosphate and 1,25-dihydroxyvitamin D3 homeostasis [7], lead to phosphate wasting and reabsorbing less [8]. Furthermore, FGF23 increased degradation of 1,25(OH)2 vitamin $\mathrm{D}$ and impaired synthesis, by means of hydroxylase enzyme [9].

At present, it has been observed that mutations in exon 22 are more common, and the mutation types in different regions are also different. In our study, a novo missense variant c.1256G > A in exon 11 was identified. The result of SIFT software showing this variant should be thought as deleterious. The symptomatic patients all carry on this variant, and the healthy members did not. The Glycine in this site among 7 species is very conserved, the $\mathrm{c} .1256 \mathrm{G}>\mathrm{A}$ variant is likely to be pathogenic (PM2, PP1, PP3-5) according to ACMG standards [10].

The XLH can't be cured and only be alleviated with drugs or surgery. So it is necessary to prevent XLH occur again, molecular genetic testing could help finding the pathological variants, and prenatal diagnosis for pregnancies is essential to prevent disease occurrence if the pathogenic variant in PHEX gene has been identified. Therefore, for the fertility requirements of the proband, preimplantation diagnosis can be suggested.

\section{Conclusions}

In conclusion, our study reported on a pedigree with XLH, also found a novo variant (c. $1256 \mathrm{G}>\mathrm{A})$ in the family. Based on the evidence concerning the correlations between the specific variant and XLH in the family, we think the mutation is responsible for $\mathrm{XLH}$.

\section{Declarations}


Acknowledgements

Not applicable.

\section{Authors' contributions}

All authors contributed to the study conception and design. Material preparation, data collection and analysis were performed by PC and XZ. The first draft of the manuscript was written by PC and all authors commented on previous versions of the manuscript. All authors read and approved the final manuscript.

\section{Funding}

This study was supported by a research grant from Jiangsu Provincial Medical Youth Talent (QNRC2016030).

\section{Availability of data and materials}

The datasets used and/or analyzed during the current study are available from the corresponding author on reasonable request.

\section{Ethics approval and consent to participate}

This study was approved by the Ethics Committee of the Drum Tower Hospital, Nanjing University (Jiangsu, China). Prior to start of this study patients provided information written consent for their volunteer contribution to this study and provided their clinical data for academic publications.

\section{Consent for publication}

All patients in this report provided their consent for publication.

\section{Competing interests}

The authors declare that they have no competing interests.

\section{References}

1. Carpenter TO. New perspectives on the biology and treatment of X-linked hypophosphatemic rickets. Pediatr Clin N Am.1997;44:443-66.

2. Sabbagh Y, Tenenhouse HS, Econs MJ, Auricchio A. Mendelian hypophosphatemias. In: Valle D, Beaudet AL, Vogelstein B, Kinzler KW, Antonarakis SE, Ballabio A, Gibson K, Mitchell G. The Online Metabolic and Molecular Bases of Inherited Disease (OMMBID).New York, NY. McGraw-Hill, 2014;Chap 197. 
3. Francis F, Hennig S, Korn B, Reinhardt R, De Jong P, Poustka A, Lehrach H, Rowe PSN, Goulding JN, Summerfield T. A gene (PEX) with homologies to endopeptidases is mutated in patients with X-linked hypophosphatemic rickets. The HYP Consortium. Nat Genet.1995;11:130-136.

4. Wei Li, Lingfang Tan, Xin Li, Xiaoyu Zhang, Xiaoyan Wu, Hongbo Chen, Lihua Hu, Xiaobei Wang, Xiaoping Luo, Fan Wang, Chengqi Xu, Qiuyun Chen, Runming Jin, Qing K. Wang. Identification of a p.Trp403*nonsense variant in PHEX causing X-linked hypophosphatemia by inhibiting p38 MAPK signaling. Human Mutation.2019;1-7.

5. Beck-Nielsen SS, Brusgaard K, Rasmussen LM, Brixen K, Brock-Jacobsen B, Poulsen MR, Vestergaard P, Ralston SH, Albagha OM, Poulsen S, Haubek D, Gjørup H, Hintze H, Andersen MG, Heickendorff L, Hjelmborg J, Gram J. Phenotype presentation of hypophosphatemic rickets in adults. Calcif Tissue Int.2010, 87:108-119.

6. Jonsson KB, Zahradnik R, Larsson T, White KE, Sugimoto T, Imanishi Y, Yamamoto T, Hampson G, Koshiyama H, Ljunggren O, Oba K, Yang IM, Miyauchi A, Econs MJ, Lavigne J, Jüppner H. Fibroblast growth factor 23 in oncogenic osteomalacia and X-linked hypophosphatemia. N Engl J Med.2003,348:1656-63.

7. White, K. E., Evans, W. E., O'Riordan, J. L. H., Speer, M. C., Econs, M. J., Lorenz-Depiereux, B., \& Strom, T. M. Autosomal dominant hypophosphataemic rickets is associated with mutations in FGF23. Nature Genetics.2000,26:345-348.

8. Gattineni J, Bates C, Twombley K, Dwarakanath V, Robinson ML, Goetz R, Mohammadi M, Baum M. FGF23 decreases renal NaPi-2a and $\mathrm{NaPi}-2 \mathrm{c}$ expression and induces hypophosphatemia in vivo predominantly via FGF receptor 1. AmJ Physiol Renal Physiol.2009, 297: F282-91.

9. Shimada T, Hasegawa H, Yamazaki Y, Muto T, Hino R, Takeuchi Y, Fujita T, Nakahara K, Fukumoto S, Yamashita T. FGF-23 is a potent regulator of vitamin D metabolism and phosphate homeostasis.J Bone Miner Res.2004,19:429-35.

10. Richards S, Aziz N, Bale S, Bick D, Das S, Gastier-Foster J, Grody WW, Hegde M, Lyon E, Spector E, Voelkerding K, Rehm HL; ACMG Laboratory Quality Assurance Committee. Standards and guidelines for the interpretation of sequence variants: a joint consensus recommendation of the American College of Medical Genetics and Genomics and the Association for Molecular Pathology. Genet Med.2015, 17:405-24.

\section{Figures}



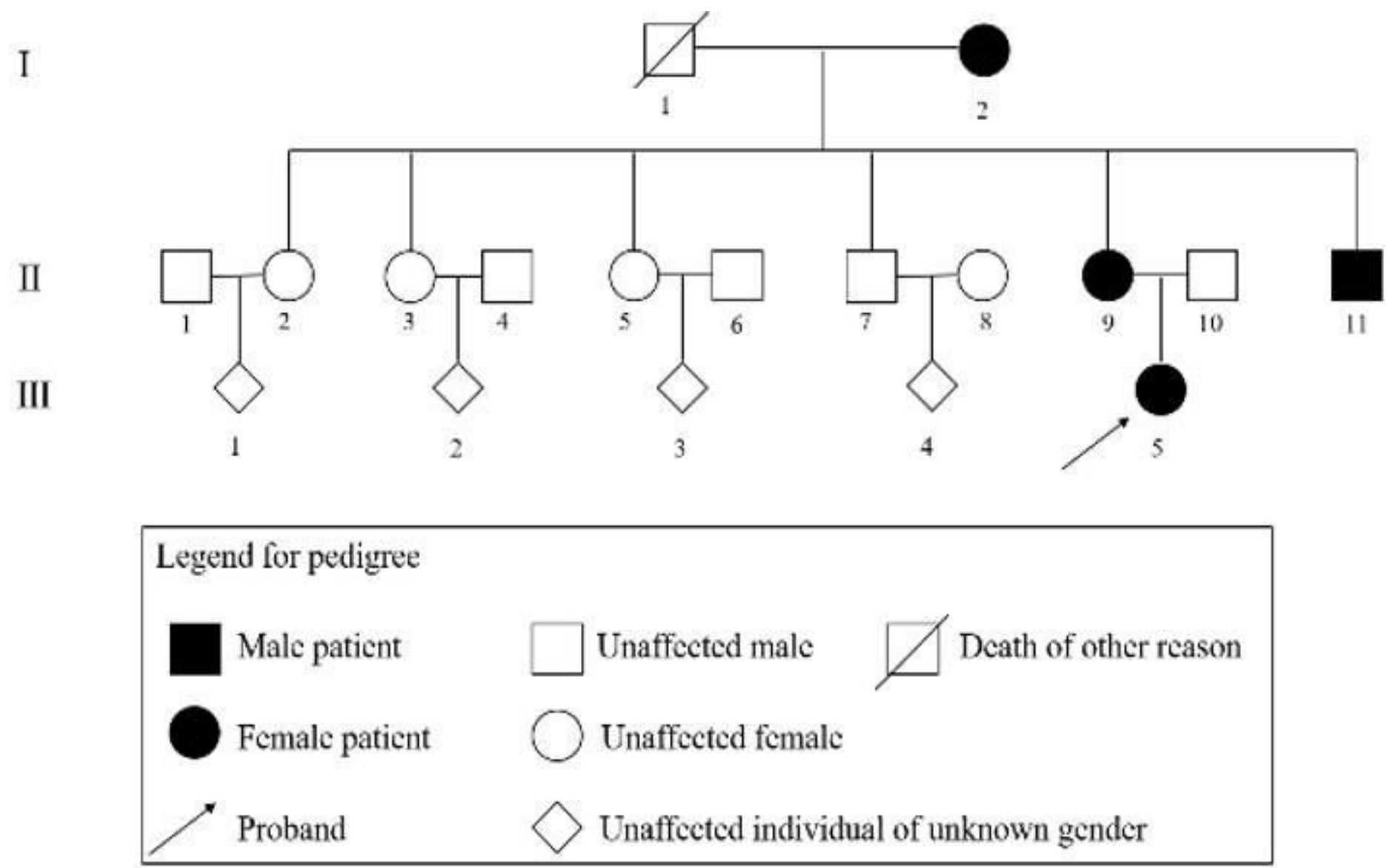

Figure 1

Pedigree of a family with X-linked hypophosphatemia.

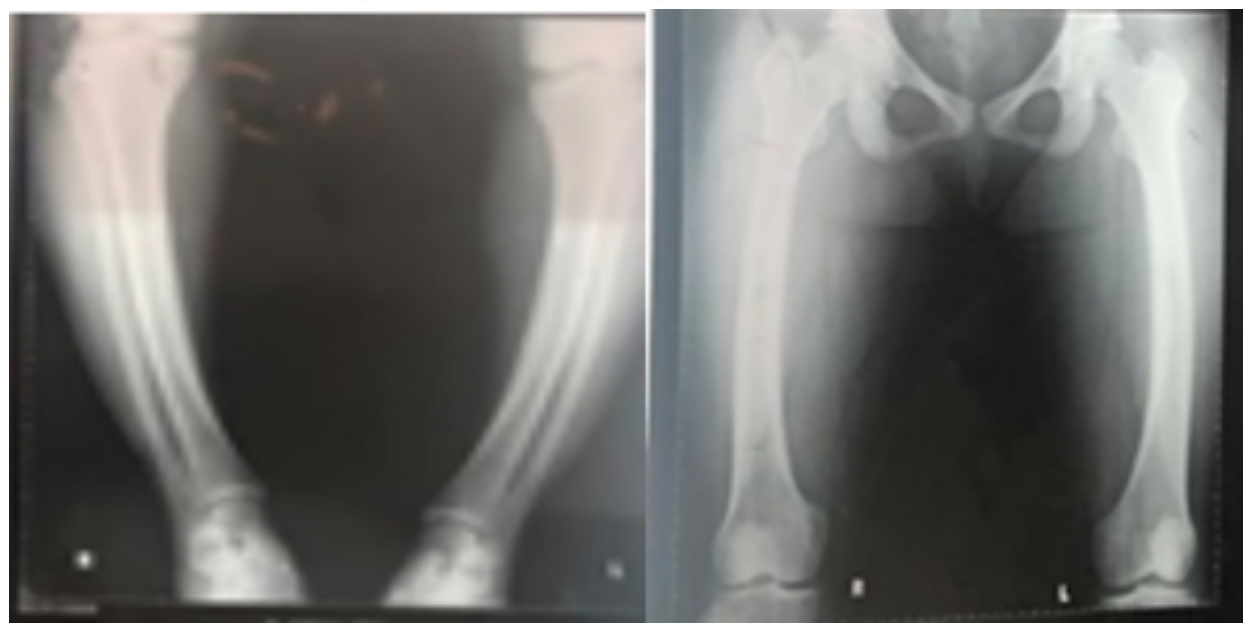

Figure 2

X-ray images of proband. X-ray of the proband showed bilateral bowed femur, tibia and fibula. 


\begin{tabular}{|c|c|c|}
\hline species & match & aa alignment \\
\hline Human & & 419 NF IES ALPYVVGKMFVDVYFQEDK \\
\hline ated & not conserved & $419 \mathrm{NF}$ I E S A L PYVVEKKMF V D VYF QED \\
\hline oglodytes & all identical & 419 NF I ES A LPYVYGKMFVDVYFQED \\
\hline & all identical & 419 NF I ESA L PYV \\
\hline culus & all identical & 419 NF I E S A L PYVYG G MF VNVHF QED \\
\hline 111us & all identical & 417 DLVESALPYVVGKMFVKAHFQED \\
\hline bripes & all identical & 434 NYVDNSLAYYTGR IF VDKHFQED \\
\hline rio & all identical & 415 NYVENTLIYAAg R FVDKHF \\
\hline anogaster & all identical & 525 EWTNKKL GVAVG GLFIRDNFNQE \\
\hline
\end{tabular}

Figure 3

Conserved amino acid at the protein product of the PHEX gene at the variant site c.1256G $>$ A in different species. The red square at this variant site showed highly conservation among 7 species. 


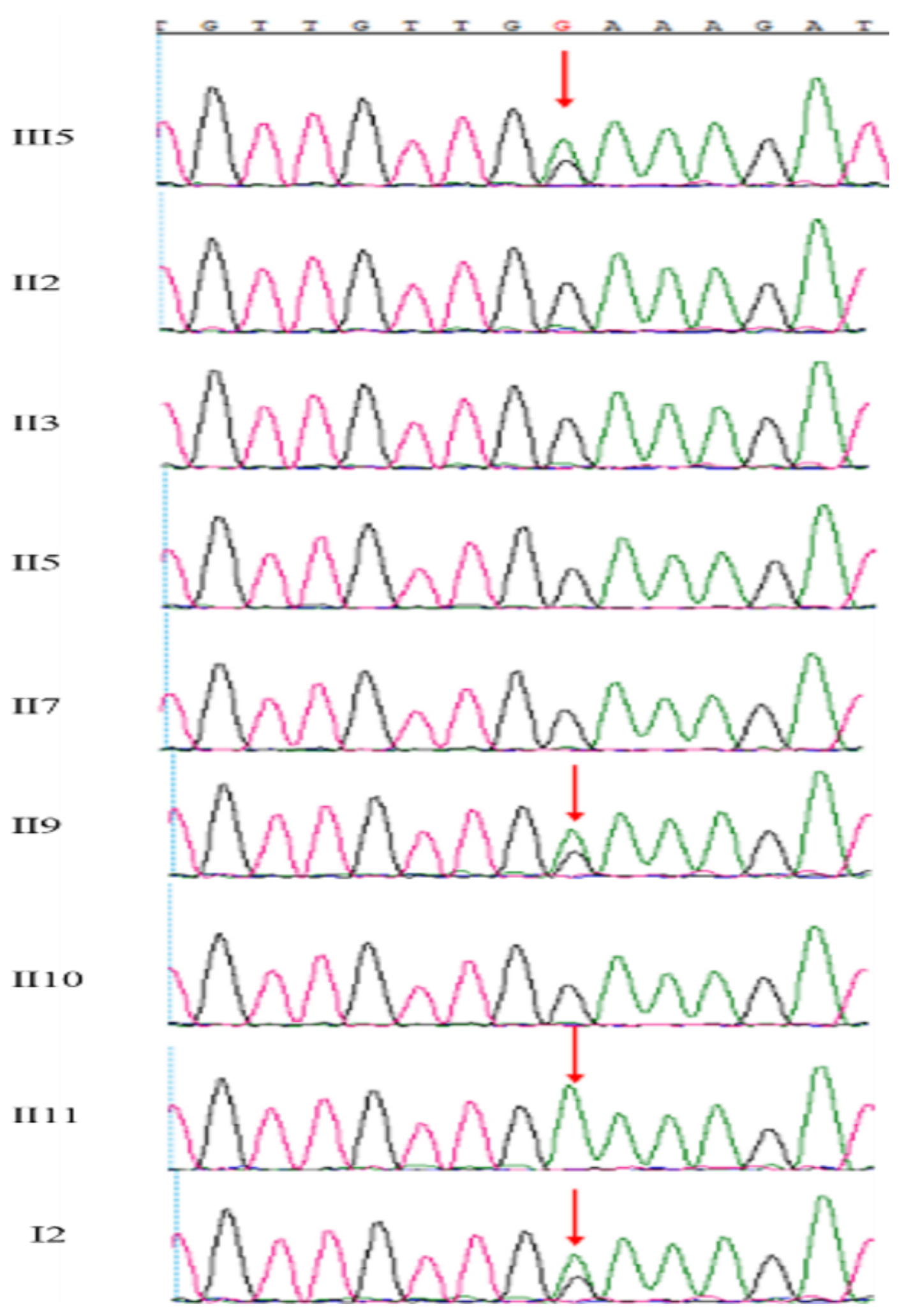

Figure 4

Regions of sequencing maps of the PHEX gene in the pedigree. Red arrows indicate variant site, NM_000444.5: c.1256G>A in the family. 